\title{
Tecnologias militares emergentes: digitalização e a Third Offset Strategy estadunidense
}

\author{
Emerging military technologies: \\ digitization and the American third offset strategy
}

Rev. Bras. Est. Def. v. 6, nº 1, jan./jun. 2019, p. 109-138

DOI: 10.26792/RBED.v6n1.2019.75118

ISSN 2358-3932

THIAGO BORNE

\section{INTRODUÇÃO}

Tentativas de compreender a relação entre tecnologia e guerra são abundantes na literatura. Em geral, os trabalhos partem de análises históricas a fim de averiguar o impacto que a introdução de diferentes tecnologias gerou na guerra ou nas forças armadas, tanto dentro quanto fora do campo de batalha. ${ }^{1}$ Em "Technology and War", por exemplo, Martin Van Creveld (1991) parte de uma premissa simples: a tecnologia governa a guerra. Neste sentido, todo o universo que permeia o conflito militar está sujeito a transformações causadas pela tecnologia: causas e objetivos; planejamento, execução e avaliação; estratégia e operações; logística; cadeias de comando e controle; etc.

Alguns autores mais contemporâneos, como Alex Roland (2016), reafirmam a ideia de Van Creveld (1991) ao assumir que a tecnologia modificou a guerra mais do que qualquer outra variável na história. Segundo ele, ainda que a prática da guerra também tenha sido largamente influenciada por fatores políticos, econômicos, ideológicos, culturais, táticos, estratégicos, psicológicos, entre outros, nenhuma destas variáveis é capaz de explicar a evolução da prática da guerra tão adequadamente quanto a tecnologia. ${ }^{2}$

Apesar de todos os avanços tecnológicos registrados ao longo da história, parece haver certo consenso na literatura de que a Segunda Guerra

Thiago Borne - Doutor em Estudos Estratégicos Internacionais pela Universidade Federal do Rio Grande do Sul (UFRGS). Bacharel em Relações Internacionais e mestre em Ciência Política pela mesma instituição. Pesquisador do Centro de Estudos Internacionais sobre Governo (CEGOV) da UFRGS e do Grupo de Estudos em Capacidade Estatal, Segurança e Defesa (GECAP) da Universidade Federal de Santa Maria (UFSM). Professor de Relações Internacionais da Universidade do Vale do Taquari (Univates) e da Escola Superior de Propaganda e Marketing (ESPM-Sul). 
Mundial deixou legados importantes para a prática da guerra contemporânea. A busca por supremacia nuclear, por exemplo, tanto por parte dos Estados Unidos quanto por parte da União Soviética, durante a Guerra Fria, não apenas orientou as relações internacionais, moldando as possibilidades de conflito internacional, mas também agiu como um dos principais vetores de desenvolvimento tecnológico na segunda metade do século XX. Foi a partir do desenvolvimento de plataformas para o lançamento de artefatos nucleares que passaram a ser desenvolvidas capacidades espaciais em ambos os países.

Considera-se, contudo, para os fins deste trabalho, que a grande contribuição da Segunda Guerra Mundial foi o desenvolvimento da computação e, consequentemente, a inauguração de um novo período histórico. Van Creveld (1991) chama este período de Era da Automação. Sua taxonomia está baseada na percepção de que, a partir de meados do século XX, a transmissão, o processamento e o armazenamento de dados passaram a ser o principal desafio das forças armadas. Este desafio, por sua vez, demandou a criação de sistemas automatizados que acabaram por substituir parte da mão-de-obra empregada nestas funções até então. Este período recebeu diferentes nomes ao longo dos últimos sessenta anos, sendo os mais conhecidos Era da Informação e Era Digital. Ambos os conceitos foram desenvolvidos com o objetivo de caracterizar de forma mais ampla os impactos de tecnologias de informação e comunicação (TIC) não apenas no âmbito militar, mas nas relações sociais como um todo.

Qualquer que seja a denominação adotada, não há dúvida de que o período contemporâneo está caracterizado pela "manipulação, armazenamento e propagação de informações em formato digital através de dispositivos eletrônicos, o que permitiu o desenvolvimento da computação digital” e de uma série de subprodutos necessários para a viabilização da comunicação entre computadores, inclusive a Internet (Canabarro 2014, 49). Esse processo pode ser chamado, grosso modo, de digitalização.

Com o final da Guerra Fria, a digitalização das forças armadas se tornou a ordem do dia nos Estados Unidos. Desde então, o país vem investindo no desenvolvimento de uma série de vetores tecnológicos que prometem preservar sua supremacia militar e manter o seu papel de destaque nas relações internacionais nas próximas décadas. O presente artigo tem caráter exploratório. Nesse sentido, busca identificar esses vetores e analisar as possíveis implicações que seu desenvolvimento e adoção poderão trazer para as forças armadas norte-americanas. Para tanto, o trabalho parte de uma revisão da literatura, na primeira seção, a partir da qual retoma o processo de digitalização das forças armadas estadunidenses. Em seguida, na segunda seção, o texto introduz o processo pelo qual os Estados Unidos 
vêm tentando impedir que outras potências tomem seu lugar como primeira potência militar, especialmente a partir do desenvolvimento tecnológico, a chamada third offset strategy. A terceira seção analisa cada um de seus vetores tecnológicos: ciber; big data; nanotecnologia; armas de energia direta; sistemas espaciais; melhoramento humano; e robótica. Finalmente, a conclusão retoma os aspectos fundamentais da pesquisa e reforça a centralidade de TIC para o planejamento de defesa contemporâneo.

\section{A DIGITALIZAÇÃO DAS FORÇAS ARMADAS DESDE A SEGUNDA GUERRA MUNDIAL}

Segundo Martin Van Creveld (1991), a possibilidade de antever e influenciar os rumos do progresso tecnológico a partir da Segunda Guerra Mundial adicionou complexidade inédita à prática da guerra. A partir do advento dos primeiros computadores, tanto os equipamentos e sistemas militares, quanto as cadeias logísticas, de treinamento e de manutenção, tornaram-se mais complexas. Nesse contexto, as instituições também tiveram de se adaptar para lidar com a necessidade de produzir, coordenar, transmitir e estocar informação em ritmo e volume crescentes. $\mathrm{O}$ desenvolvimento da computação permitiu que boa parte destas tarefas fosse realizada de forma automatizada. Apesar disso, se por um lado o processo garantiu relativo ganho de eficiência na administração militar, por outro, acelerou ainda mais o ritmo e volume de dados produzidos. Em outras palavras, a partir de então, mais computadores ${ }^{3}$ gerariam mais dados, aumentando a demanda por ainda mais computadores e assim sucessivamente. ${ }^{4}$

Ao final da Guerra Fria, os exércitos passaram a se reestruturar ao redor do mundo. $\mathrm{O}$ fim da União Soviética não apenas justificou cortes orçamentários e reduções de pessoal, mas também catalisou processos de privatização e de terceirização das forças armadas em muitos países. ${ }^{5}$ Nos Estados Unidos, estes processos foram reforçados também por uma crescente aversão da população a guerras. As Guerras do Vietnã (1954-1975) e da Coréia (1950-1953), principalmente, deixaram marcas profundas na população, que passou a questionar ativamente a necessidade de mandar jovens cidadãos para lutar no exterior. ${ }^{6}$ Nesse contexto, o planejamento militar norte-americano passou a considerar novos cenários para o emprego da força, marcados pela complexidade e pela assimetria. Além disso, na ausência de um competidor direto, os Estados Unidos vieram a consolidar a sua proeminência no planeta assumindo uma série de novas responsabilidades no plano internacional, tais como a condução de missões de paz, de intervenções humanitárias e de operações contraterrorismo (Gunzinger 2013; Stulberg and Salomone 2007). 
Este movimento de reforma foi fortemente marcado pelas TIC características da Era Digital e traduzido pelo caráter estruturante que a ideia de revolução nos assuntos militares (RMA) teve para o Departamento de Defesa dos Estados Unidos. Segundo Duarte (2012), a ideia de RMA desenvolveu-se em dois momentos históricos distintos. O primeiro diz respeito às reformas estruturais e doutrinárias colocadas em curso frente ao surgimento das armas nucleares durante a Guerra Fria, especialmente na União Soviética. O segundo momento está atrelado ao emprego de TIC durante a Guerra do Golfo (1990-1991) e a uma conformação essencialmente norte-americana de reforma de suas forças armadas. Nas palavras do autor:

Esta seria a resposta a duas demandas que justaporiam um dilema de projeto de força: a redução iminente das dimensões das forças armadas [causada] pelo fim da Guerra Fria; e a sustentação de uma estrutura de capacidades combatentes elevadas em um ambiente de incerteza e de ambiguidades de cenários de ameaça e confronto (Duarte 2012, 9).

No cerne da RMA estadunidense está a ideia de que:

A combinação entre ataques aéreos precisos e ataques por mísseis dominaria a guerra futura e que a disputa pela supremacia de informação substituiria as batalhas de ruptura como o aspecto dominante para o sucesso. Os desproporcionais e infalíveis meios de combate à distância garantiriam que o grande obstáculo se tornaria apenas a identificação dos alvos, ao que se seguiria sua rápida comunicação para as plataformas e vetores disponíveis (Duarte 2012, 9).

Por isso, o foco do processo estaria, pela primeira vez, no componente lógico - o software - do processo de transmissão de dados. Ou, ainda, em uma "mudança da plataforma [o hardware] para rede" (Cebrowski and Garstka, 1998) — uma afirmação questionável, já que redes dependem necessariamente de hardware para o seu funcionamento. Importava, neste sentido, transformar as forças armadas norte-americanas, desenvolvendo o "sistema dos sistemas". Após a vitória no Golfo, onde os Estados Unidos enfrentaram um oponente incapaz de compreender as vicissitudes de sua própria força e de seu ambiente operacional, a ideia de "transformação" (transformation) foi avançada como plataforma política para as reformas militares do país.

A RMA foi objeto de estudo e debate político constante ao longo dos anos 1990 e início dos 2000, e suscitou o surgimento de abordagens concorrentes para o fenômeno da digitalização. Análises detalhadas de cada uma delas podem ser encontradas em Cohen (1999) e Duarte (2012). Não 
obstante a dificuldade de conceitualizar a RMA, a mesma acabou traduzindo-se em política por meio da já mencionada "transformação" do aparato militar norte-americano. Segundo Duarte $(2012,20)$ a transformação:

Propunha o desenvolvimento de uma capacidade de detecção, antecipação e controle de ondas de RMA de maneira a dar aos Estados Unidos, ao mesmo tempo, a vantagem militar absoluta, a partir da produção de novos sistemas de armamentos sem equiparação com outros países, e comparativa, a partir de processos mais eficientes de produção de armamentos e manutenção de tropas. Assim, a "transformation" exploraria os efeitos táticos e estratégicos da tecnologia militar, mas também reduziria os custos da guerra, tornando-a um empreendimento muito mais razoável para os Estados Unidos. Suas forças armadas seriam providas de equipamentos e armamentos muito mais letais e precisos, bem como passariam a contar com uma capacidade logística para sustentar suas operações por muito mais tempo do que qualquer potência concorrente. Desta maneira, os Estados Unidos poderiam explorar uma primazia política mundial por muito tempo, prolongando seu status de potência unipolar.

O processo foi comandado pelo então Secretário de Defesa Donald Rumsfeld no final dos anos 1990 e colocado à prova no Afeganistão e no Iraque após os atentados terroristas de 2001. Naquele momento, esperava-se que os esforços americanos obtivessem o mesmo sucesso que tiveram no Golfo, dez anos antes. Infelizmente, os oponentes dos Estados Unidos passaram a lutar de forma diferente a fim de anular a superioridade tecnológica norte-americana. Neste sentido, passaram a combater entre civis em áreas urbanas e populosas, e a recorrer à insurgência e ao terrorismo. A esta dificuldade tática soma-se a instabilidade de sistemas de alta tecnologia em ambientes extremos. No deserto, muitos equipamentos paravam de funcionar devido ao calor e à areia. Além disso, o uso exagerado de TIC acabou gerando novas - e muitas vezes inesperadas - demandas no fronte. Suprir a necessidade crescente por fontes de energia, por exemplo, tornou-se um problema logístico para as forças da coalizão (Singer 2009).

Nesse sentido, a "belle époque" (Arrighi 2008) norte-americana dos anos 1990-2000 foi caracterizada pela tentativa de proporcionar informação quase-perfeita para o combatente por meio da introdução do conceito de guerra centrada em redes. A chave deste conceito estava justamente na adoção de TIC - computadores, o Global Positioning System (GPS), linhas de comunicação e de troca de dados dedicadas, montadas com cabos de fibra ótica e tecnologias wireless de alta velocidade, e, mais recentemente, a própria Internet - para alcançar níveis avançados de conectividade e partilha de informação entre as unidades. ${ }^{7}$ A organização das unidades em 
redes descentralizadas e distribuídas, por sua vez, permitiria ao comandante e às tropas o controle total do teatro de operações. Assim, o domínio informacional proporcionado pela inovação tecnológica eliminaria as incertezas e ambiguidades das operações. Em outras palavras, a digitalização resultante, grosso modo, do emprego de redes de computadores extinguiria a névoa responsável por "deixar difícil até o mais simples da guerra".

\section{A THIRD OFFSET STRATEGY ESTADUNIDENSE}

O relativo fracasso das operações militares dos Estados Unidos no início do século XXI, notadamente no Afeganistão e no Iraque, evidenciou que nem mesmo o uso avançado de TIC é capaz de modificar a natureza fundamental da guerra. A despeito das críticas, ela ainda é um ato político violento, marcado pela confusão e pela incerteza, pela névoa e pela fricção. É inegável, no entanto, que TIC podem ajudar a minimizar seus efeitos, aumentando a coesão e a consciência situacional a níveis inéditos na história.

Em que pese as lições aprendidas recentemente no Oriente Médio, a RMA, apesar de ter perdido força política, parece não ter sido completamente esquecida (Lawson 2010). Pelo contrário, a ideia de transformar os Estados Unidos na única potência capaz de orientar o caráter futuro da guerra (e, portanto, vencê-la sob quaisquer circunstâncias) continua vigente. $\mathrm{O}$ que se viu no início deste século foi justamente a tentativa de os Estados Unidos transformarem a guerra exatamente naquilo que gostariam que fosse. Uma vez que as expectativas estadunidenses não foram concretizadas, coube ao estamento político-militar buscar alternativas. É nesse contexto - que previa, ainda, cortes futuros no orçamento militar - que começa a ser debatida a third offset strategy.

De maneira concisa, a third offset strategy é "uma tentativa de contrapor o encolhimento da estrutura da força militar estadunidense e de sua superioridade tecnológica declinante em uma era de competição entre grandes potências" (Eaglen 2016). Soma-se a isso, a necessidade de lidar com os imperativos de guerras irregulares a partir de uma solução capaz de tratar de ambos os problemas simultaneamente. A estratégia diz respeito, nesse contexto, tanto à aquisição de novas tecnologias quanto à manutenção das capacidades dissuasórias tradicionais dos Estados Unidos. Ou, em outras palavras:

A third offset não é apenas uma busca pelas tecnologias da próxima geração, mas também uma reavaliação dos programas existentes com um foco sobre como eles podem ser dramaticamente melhorados com custos relativamente baixos. O objetivo dos oficiais de defesa é explorar as vantagens duradouras dos Estados Unidos e impor custos aos inimigos ou adversários potenciais" (Eaglen 2016, tradução nossa). 
Não é a primeira vez que o país passa por um processo de "offset". O primeiro se deu no início dos anos 1950, logo após a Segunda Guerra Mundial. Seu objetivo era anular a influência soviética sobre a Europa Oriental por meio do desenvolvimento de capacidades nucleares superiores. O segundo processo de "offset" ocorreu entre os anos 1970 e 1980, e baseou-se nas tecnologias que moldaram o conceito de RMA.

Um ponto central da discussão sobre a third offset é justamente quais capacidades adversárias a estratégia pretende compensar. Ambas as estratégias anteriores estiveram focadas na compensação da vantagem numérica que a União Soviética possuía em relação aos Estados Unidos em termos de capacidades tradicionais. Atualmente, contudo, a a erosão da vantagem tecnológica dos Estados Unidos não deriva da vantagem numérica ou do volume superior de investimentos dos adversários, mas da "natureza crescentemente global e comercial do ambiente de inovação, e da crescente aplicabilidade de tecnologias comerciais em operações militares" (Ellman, Samp, and Coll 2017, 2).

No contexto da Era Digital, o crescimento exponencial na capacidade de processamento de dados computacionais tem sido um fator determinante para o desenvolvimento de muitas tecnologias militares emergentes. ${ }^{8}$ Hoje em dia, esse desenvolvimento tecnológico é capitaneado, em grande medida, por empresas privadas, sinalizando uma mudança em relação ao passado, quando boa parte dele era patrocinado pelo Estado. Ainda assim, a possibilidade de ampliar o escopo de aplicação original de tecnologias civis, adaptando-as para o emprego na guerra, tem despertado o interesse de diversos entes estatais e fomentado uma aproximação maior entre as esferas pública e privada.

Nesse sentido, tanto a academia quanto militares, políticos e empresários têm convergido em relação a alguns vetores tecnológicos que serão cruciais para as forças armadas nas próximas décadas. ${ }^{9}$ Por trás dessa convergência estão pelo menos duas premissas referentes ao caráter da guerra no século XXI. A primeira é a prevalência de guerras irregulares complexas $^{10}$ sobre guerras interestatais tradicionais e a centralidade de capacidade de $\mathrm{A}_{2} \mathrm{AD}^{11}$ (anti-acesso e negação de área) e sistemas computadorizados de C3 $\mathrm{I}^{12}$ (comando, controle, comunicação e inteligência) nesse contexto. A segunda é a ideia de que diferentes tecnologias podem atuar como multiplicadores de força importantes nos ambientes tático e estratégico definidos pela primeira premissa. ${ }^{13}$ Ao definir essas premissas, busca-se compreender a conjuntura atual, projetando suas consequências para o futuro. Desta sorte, é possível não apenas avaliar corretamente as demandas das guerras contemporâneas, mas também dissuadir a eclosão de conflitos futuros. A third offset tenta, em última instância, avaliar onde, contra quem, pelo que e como serão as próximas guerras. 


\section{TECNOLOGIAS MILITARES EMERGENTES}

É possível agregar os vetores tecnológicos que integram a segunda premissa em pelo menos sete categorias, muitas das quais se confundem com aquelas formalmente presentes na third offset strategy dos Estados Unidos: ciber; big data; nanotecnologia; armas de energia direta; sistemas espaciais; melhoramento humano; e robótica. ${ }^{14}$ É interessante notar que todas dependem da computação digital para se desenvolver (Wong 2013). Nesse sentido, estão intimamente relacionadas. Apesar dessas tecnologias já serem, em alguma medida, aplicadas em campo de batalha, o seu emprego ainda suscita uma série de questionamentos de cunho ético e moral, bem como desafia os ordenamentos jurídicos e políticos atualmente vigentes. Os próximos parágrafos se dedicam a apresentar apenas uma visão geral dos tópicos, indicando leituras pertinentes para cada um deles. O objetivo do texto não é, portanto, exauri-los, mas demonstrar como a amplitude dos temas permite a condução de pesquisas individuais.

\section{Ciber e a Internet das Coisas}

A Internet é o principal fruto da Era Digital. Nenhuma outra tecnologia modificou tantos aspectos das relações sociais em tão pouco tempo. Hoje em dia, é difícil pensar em alguma área que não seja afetada por níveis crescentes de conectividade. ${ }^{15} \mathrm{O}$ conceito de Internet das Coisas (Internet of Things) surge justamente nesse contexto de proliferação de aparelhos capazes de acessar a Rede e "conversar" entre si. Neste sentido, gerenciar o crescente volume de dados proveniente deste ecossistema complexo tornou-se um desafio para as forças armadas, ${ }^{16}$ que passaram a ter de considerar a manutenção da segurança de inúmeros dispositivos e da informação gerada por eles.

Ainda que a correlação entre guerra e ciberespaço seja explorada pela literatura desde os anos 1990 - sendo o trabalho de Arquilla e Rondfeldt (1997) um dos pioneiros neste sentido - , a partir de meados dos anos 2000, casos como o da Estônia (2007), da Guerra da Ossétia do Sul (2008), e do Stuxnet (2010) passaram a ser exaustivamente estudados e culminaram na criação de uma área específica para os estudos de cibersegurança (Libicki 2012; Rid 2013). A importância do campo foi reafirmada em 2013, graças ao apelo do Caso Snowden, quando os vocábulos "ciberinteligência”, "ciberespionagem" e "cibersabotagem" passaram a integrar definitivamente os léxicos de defesa.

Nesse contexto, as forças armadas de diversos países passaram a estabelecer instituições voltadas exclusivamente para o estudo e prática da 
defesa e da inteligência cibernética. Brasil e Estados Unidos, por exemplo, criaram o Centro de Defesa Cibernética do Exército (CD Ciber) e o Comando Cibernético (USCYBERCOM), respectivamente, sinalizando uma maior disposição e organização de esforços por parte dos Estados para se prepararem para uma eventual guerra cibernética.

Apesar disso, a possibilidade de ocorrência de uma guerra cibernética ainda é bastante controversa na literatura. Enquanto alguns autores chamam atenção para os perigos de um suposto "Ciber Pearl Harbor", outros mais conservadores tendem a questionar o potencial destrutivo de ações cibernéticas. No que tange a guerra, mais recentemente, a literatura tem se focado em questões relativas à atribuição, à relação ataque-defesa, à inovação, entre outros. Ainda assim, cada vez mais o prefixo "ciber" tem sido utilizado para caracterizar também outros tópicos recorrentes nos Estudos Estratégicos e nas Relações Internacionais, sinalizando uma atualização/ ampliação das agendas de pesquisa mais tradicionais desses campos no contexto da Era Digital.

\section{Big Data}

Desde os atentados terroristas de 2001, e em virtude da concretização de uma realidade projetada para incrementar cada vez mais a possibilidade de interconexão de pessoas e dispositivos, a quantidade de dados recolhidos pela inteligência norte-americana aumentou cerca de $1.600 \%$, segundo reportagem de 2012 (Young 2012). Da mesma forma, as forças armadas estão operando com um volume de informação sem precedentes históricos. O termo "big data" diz respeito justamente a essas grandes quantidades de dados, cujo processamento não pode ser realizado com ferramentas tradicionais. Posto de forma mais estruturada, big data refere-se à emergência de " $[\ldots]$ new datasets with massive volume that change at a rapid pace, are very complex, and exceed the reach of commonly used hardware environments and software tools for data management" (Akhgar 2015, 3). A ideia, portanto, é que grandes volumes de dados são capazes de gerar informação que provavelmente seria ignorada caso fossem consideradas amostras pequenas.

Além disso, o termo refere-se ainda à possibilidade de transformar em dados aspectos do mundo que jamais foram quantificados, em virtude da possibilidade de implementar microcomputadores e sensores em um sem-número de seres e dispositivos. Kenneth Neil Cukier e Viktor MayerSchoenberger (2013) chamam esse processo de "datafication":

location has been datafied, first with the invention of longitude and latitude, and more recently with GPS satellite systems. Words 
are treated as data when computers mine centuries' worth of books. Even friendships and "likes" are datafied, via Facebook.

O processo difere de abordagens mais tradicionais não apenas porque lida com volumes maiores de dados, mas também porque reconhece, neste grande universo, a validade de amostras mais toscas. Ademais, permite ainda que sejam apontadas correlações que não são necessariamente causais. Por isso, tomando novamente as palavras de Cukier e Mayer-Schoenberger (2013),

big data might change our way of thinking about the world. As we tap ever more data to understand events and make decisions, we are likely to discover that many aspects of life are probabilistic, rather than certain.

O conceito não é necessariamente novo, e está presente na literatura desde pelo menos o início dos anos 2000, mas popularizou-se com o aprofundamento da Internet das Coisas. Além disto, as revelações de Edward Snowden em 2013 ajudaram a fomentar o debate internacional sobre o tema, ao expor a capacidade de os Estados Unidos explorarem praticamente todos os componentes tecnológicos que conformam as redes de comunicação ao redor do mundo para coletar e acumular informação. ${ }^{17} \mathrm{~A}$ partir de então, cada vez mais países passaram a questionar o que fazer com este volume de dados crescente e como aplicar a big data para gestão pública. Ao mesmo tempo, lidar com este montante crescente de dados passou a ser visto como um desafio para as forças armadas e serviços de inteligência, na medida em que demanda não apenas capacidades de transferência e armazenamento, mas também de análise. Dados precisam ser tratados de maneira sistemática para que se transformem em informação — dados estruturados - relevante para os processos decisórios.

Neste contexto, as forças armadas e os serviços de inteligência foram bastante afetados. Por um lado, a noção de big data promete integrar e organizar grandes quantidades de informação, permitindo a sua aplicação a uma extensa gama de funções, tais como análise de redes sociais e modelagem de sistemas de armas. Além disso, de modo mais geral, "big data analytics reduces the processing time of a query and in turn reduces the time to wait for the solutions. Combining and analyzing the data allows data-driven (directed) decision making" (Akhgar 2015, 8), o que, por sua vez, auxilia o desenvolvimento de ações significativas no tempo e espaço ideais. Por outro lado, a realização destas capacidades depende de uma série de fatores técnicos referentes, por exemplo, ao pareamento de dados oriundos de fontes e com formatos distintos. ${ }^{18}$ Somam-se a estes desafios outros de caráter político — ou de governança - como o estabelecimento 
de medidas de segurança robustas o suficiente para evitar o vazamento ou a exploração dos dados armazenados por atores não-autorizados (Symon and Tarapore 2015). Por isto, explorar as oportunidades e mitigar os riscos oriundos da big data requer visão estratégica e, possivelmente, o desenvolvimento de novas habilidades, novas ferramentas e novos processos de gerenciamento de pessoal por parte dos militares e agentes de inteligência.

\section{Nanotecnologia}

A nanotecnologia diz respeito ao estudo de sistemas cujo tamanho é inferior a 100 nanômetros em pelo menos uma dimensão. Um nanômetro é o equivalente a um bilionésimo de metro $\left(1 \times 10^{-9}\right)$. Por exemplo, um nanômetro está para um metro assim como o diâmetro da moeda de um centavo está para o diâmetro da Terra, grosso modo. O diâmetro de um glóbulo branco é da ordem de 10 mil nanômetros. É, portanto, o estudo da manipulação da matéria em escala atômica ou molecular. O campo é multidisciplinar e inclui o desenvolvimento de materiais ou componentes e está associado a diversas áreas de pesquisa e produção, tais como medicina, eletrônica, ciência da computação, física, química, biologia e engenharia dos materiais. O objetivo da nanotecnologia é desenvolver estruturas estáveis a partir da manipulação atômica. Em outras palavras, a nanotecnologia trata de agrupamentos de átomos ou moléculas, cujo arranjo espacial e composição são usados para obter estruturas com novas propriedades mecânicas, ópticas, eletrônicas ou magnéticas e, portanto, novos produtos industriais.

Vale ressaltar, contudo, que nanotecnologia é diferente de miniaturização. Via de regra, nanotecnologias têm propriedades ou funcionalidades diferentes de suas versões macro ou microscópicas, oriundas justamente de suas dimensões nanométricas. Os transistores de computadores, por exemplo, têm dimensões inferiores a 100 nanômetros e nem por isso são dispositivos nanotecnológicos, uma vez que suas capacidades são idênticas a de seus pares micrométricos. Partículas nanométricas de prata, por outro lado, oferecem propriedades diferentes de seus pares micrométricos e têm sido largamente usadas, por exemplo, na indústria farmacêutica:

[...] silver nanoparticles are extraordinarily efficient at absorbing and scattering of light due to its optical properties. Their strong interaction with light occurs because the conduction electrons on the metal surface undergo a collective oscillation when they are excited by light at specific wavelengths (Mavani and Shah 2013, 2).

Por ser um campo multidisciplinar, a nanotecnologia possibilita aplicações variadas. Em termos militares, os esforços têm se focado principal- 
mente no desenvolvimento de produtos farmacêuticos, sensores, têxteis e "smart materials" em geral. A combinação destes vetores busca a criação de materiais leves, resistentes e multifuncionais, capazes de proporcionar ao combatente maior proteção, mobilidade e conectividade. Dentre as ideias em desenvolvimento estão uniformes praticamente invisíveis, roupas leves que se tornam rígidas quando o soldado quebra uma perna ou braço, exoesqueletos capazes de multiplicar a força do combatente e sapatos que acumulam energia, permitindo ao soldado saltar longas distâncias (Nasu 2013; Wang 2016).

\section{Armas de energia direta}

A partir da década de 1980, com o desenvolvimento da Iniciativa Estratégica de Defesa dos Estados Unidos, armas de energia direta se tornaram pauta constante nos debates sobre novas tecnologias militares (Siniscalchi 2001). Grosso modo, a categoria compreende armas baseadas em lasers, micro-ondas de alta potência (high-power microwaves ou HPM), e plasmas. A aplicação destas tecnologias permite engajar com o inimigo em velocidade da luz ou próxima dela, e a entrega de destruição praticamente ilimitada quando comparada àquela proporcionada por armas cinéticas municiadas.

Além dos Estados Unidos, países como Reino Unido, Alemanha, França, Israel, Rússia e China estão envolvidos na manutenção de linhas de pesquisa e desenvolvimento de armas de energia direta há bastante tempo (IISS 2015). Duas áreas, mais especificamente, têm sido alvo principal destas iniciativas: sistemas laser e HPM. Os esforços da Marinha norte-americana merecem destaque neste sentido, sobretudo no desenvolvimento de sistemas laser antiaéreos voltados para neutralização de aeronaves de ataque e UAS de alta velocidade. Já na esfera das micro-ondas de alta potência, tanto o Reino Unido quanto a Rússia desenvolvem desde os anos 1990 sistemas capazes de emitir pulsos eletromagnéticos potentes o suficiente para neutralizar sistemas eletrônicos e computacionais. Não obstante avanços significativos no campo, a aplicação de HPM ainda é bastante limitada. Primeiro, dada a dificuldade de se manterem padrões constantes de emissão de energia e de se avaliar com precisão os danos causados nos sistemas inimigos. Segundo, devido à impossibilidade de se controlar efeitos de segunda e terceira ordem oriundos de sua utilização, inclusive os efeitos potencialmente danosos causados aos próprios sistemas amigos. ${ }^{19}$

Por isto, recentemente, a aplicação destes dispositivos passou por uma reavaliação que a distanciou das previsões oitentistas de Guerra nas 
Estrelas. Neste sentido, armas de energia direta passaram a ser vistas como complementos a sistemas tradicionais, ou, em outras palavras: “[ $[.$. a disruptive technology that can potentially provide substantial military beneft at the tactical rather than strategic level" (IISS 2015, 9), desde que os sistemas atinjam o grau de maturidade necessário para operarem.

\section{Sistemas espaciais}

A capacidade de desenvolver sistemas espaciais esteve exclusivamente vinculada às superpotências da Guerra Fria por muito tempo. Atualmente, contudo, o cenário é bastante diferente daquele da segunda metade do século XX. Hoje em dia, aproximadamente 56 países possuem recursos espaciais de sua propriedade ou de uso compartilhado. O número de países produtores de satélites é menor, mas ainda assim significativo, incluindo Alemanha, Canadá, China, Estados Unidos, França, India, Israel, Japão e Rússia. Por sua vez, a lista de atores com capacidade própria de lançamento de satélites congrega Brasil, China, Coreia do Norte, Coreia do Sul, Estados Unidos, Índia, Rússia, Ucrânia e União Europeia. Finalmente, apenas três países foram capazes de realizar missões tripuladas completas até hoje: Estados Unidos, Rússia e China (Cepik et al. 2015).

Aliada a esta mudança de distribuição de capacidades em escala geográfica está uma visão mais ampla da importância do espaço sideral, não mais atrelada apenas a questões militares, mas também a questões econômicas, tecnológicas e políticas. Neste sentido, o comando do espaço — ou seja, a capacidade de um país garantir por meios próprios o acesso e uso do espaço, sem que outro possa lhe negar tal acesso (Cepik and Machado 2011,114 ) - passou a ser visto como um exercício relacionado ao desenvolvimento social, à consolidação de setores industriais nacionais, à disputa pela institucionalização de regras no sistema internacional, entre outros.

Isto não significa que o espaço tenha perdido sua importância como ambiente operacional. Pelo contrário: na medida em que as forças armadas se digitalizam, sistemas espaciais se tornam cada vez mais centrais para uma série de tarefas, incluindo aquisição de alvos, monitoramento, vigilância, navegação e comunicação. Nesse sentido, os dados digitais passam não apenas a depender das linhas celestiais de comunicação, mas também da resiliência de satélites para circular. ${ }^{20} \mathrm{~A}$ fragilidade destes sistemas a danos, intencionais ou acidentais, e a possibilidade de o espaço sideral ser utilizado como meio para a entrega de artefatos nucleares, faz com que sua governança seja um assunto proeminente ainda hoje entre a comunidade internacional. ${ }^{21}$ 


\section{Melhoramento humano 22}

Esta categoria diz respeito à aplicação de tecnologias diversas para o melhoramento das capacidades físicas e/ou mentais dos combatentes. Segundo uma das definições mais difundidas, a do bioeticista Eric Juengst (1998, 29), compreende intervenções médicas ou biológicas no corpo “[ [...] to improve performance, appearance, or capability besides what is necessary to achieve, sustain or restore health". Posto de outra forma, a lógica por trás do melhoramento humano é "hackear" (Lin 2013) o corpo, modificando-o conforme as necessidades do campo de batalha ou segundo as funções que o sujeito for desempenhar. Neste sentido, a categoria está diretamente vinculada aos demais tópicos descritos nesta seção, especialmente à nanotecnologia, biotecnologia e biomedicina, genética e ao desenvolvimento de fármacos e próteses, por exemplo.

Se por um lado a ideia de melhorar as capacidades fundamentais dos seres humanos parece distante da realidade, por outro, para alguns cientistas, o processo já é bastante comum. Basta pensar, por exemplo, nos efeitos que estimulantes e drogas bastante acessíveis como a cafeína e metanfetaminas têm sobre o corpo. Na verdade, estas substâncias são amplamente utilizadas por soldados no mundo todo a fim de aumentar os seus níveis de prontidão e responsividade. Por outro lado, nem todas as drogas ou todos os dispositivos, por mais tecnológicos que sejam, podem ser considerados melhoramentos. Desta sorte, definir exatamente o que é e o que não é um melhoramento continua sendo um desafio, sobretudo no campo da saúde, já que:

The distinction between health-oriented and enhancing interventions will not always be clear, and invariably there will be borderline cases. The difficulty of clearly identifying what counts as an enhancement complicates the task of determining the conditions, if any, in which it would be ethical to research or use enhancements in the military (Lin et al. 2013, 18).

Ainda que os esforços para criar "supersoldados" tenham diminuído na década de 2000 (Hanlon 2004; Burnam-Fink 2011) nos Estados Unidos - sobretudo em virtude das demandas oriundas das guerras no Oriente Médio - , o país continua investindo no desenvolvimento destas capacidades por meio do financiamento de uma série de projetos. $\mathrm{O}$ mesmo vale para países como China e Rússia, que, segundo alguns analistas (Axe 2012), também desenvolvem programas de melhoramento humano, mas não estão sujeitos aos mesmos mecanismos de accountability que os países ocidentais. Em seu estudo, Patrick Lin et al. (2013) analisaram uma série 
de projetos de melhoramento humano atualmente em curso em diferentes países, classificando as iniciativas em quatro categorias: capacidades físicas (incluindo força, mobilidade, proteção); capacidades cognitivas (incluindo consciência, atenção, memória, aprendizagem, comunicação e linguagem); capacidades sensoriais (visão, olfato, audição, tato, paladar); e capacidades metabólicas (incluindo resistência, alimentação, sono e saúde) ${ }^{23}$ Além destas, uma quinta categoria contempla pesquisas científicas de caráter mais genérico. A partir desta classificação, os autores discutem as questões éticas e jurídicas que, em sua visão, devem guiar o desenvolvimento e a aplicação de melhoramentos para/em combatentes. Os autores concluem que, apesar de melhoramentos humanos serem potencialmente benéficos para a condução de atividades militares, estes ainda enfrentarão diversos obstáculos, tanto tecnológicos quanto políticos, até que sejam amplamente aceitos.

\section{Robótica e inteligência artificial}

É difícil indicar o momento exato em que sistemas robóticos passaram a ser considerados seriamente no planejamento militar. $\mathrm{O}$ debate sobre o seu desenvolvimento para guerra não é necessariamente novo, e remonta a pelo menos meados do século XX. Apesar disto, somente recentemente, com a popularização dos drones, o assunto tornou-se um ponto relevante para a comunidade em geral. Capitaneando este debate estão questões relativas à moralidade dos chamados "assassinatos seletivos", à necessidade de a comunidade internacional frear o desenvolvimento de "robôs assassinos" e à legalidade do emprego destes sistemas em operações militares, por exemplo. Ademais, a identificação de temas emergentes no campo da robótica indica algumas tendências interessantes relativas ao desenvolvimento de capacidades militares no futuro. Nesse contexto, destacam-se os debates relativos à autonomia, robótica de enxame e relacionamento homem-robô.

A partir dos atentados terroristas de 2001, sistemas robóticos tornaram-se um dos principais recursos dos Estados Unidos na Guerra ao Terror. Segundo dados de Peter Singer (2009, 61), entre 2002 e 2008 o orçamento de defesa anual norte-americano aumentou $74 \%$, chegando a USD 515 bilhões. O montante não inclui os gastos com as operações no Afeganistão e no Iraque, que foram custeados com valores suplementares - tampouco os montantes investidos em pesquisa e desenvolvimento e em projetos confidenciais, cujos orçamentos não são divulgados para o público. Boa parte desse dinheiro foi dirigido para sistemas robóticos. Ainda de acordo com Singer (2009, 61), desde 2001 o valor investido em sistemas terrestres tem dobrado a cada ano, enquanto os investimentos em sistemas aéreos aumentam $23 \%$ ao ano. 
Os dados deixam claro, portanto, que, apesar de o processo de robotização ocorrer entre as forças armadas estadunidenses desde o começo do século XX, ele tem se intensificado nas últimas três décadas, e atingiu seu pico nos anos 2000, graças aos esforços do país na África e no Oriente Médio. Não está claro, contudo, se ele continuará a se intensificar ou se recuará nos próximos anos. Ainda que a tendência geral seja de, no mínimo, manutenção de capacidades adquiridas, até a eleição de Donald Trump alguns analistas vinham sugerindo que os gastos militares deveriam diminuir no longo prazo (Ackerman 2013). De acordo com um relatório do Center for the Study of the Drone, do Bard College, "funding for drones is lower in the proposed Fiscal Year 2017 budget than in Fiscal Year 2016, although it is slightly higher than in Fiscal Year 2015" (Gettinger 2016). O mesmo documento também reporta um interesse crescente do Departamento de Defesa tanto em sistemas terrestres quanto em sistemas navais, refletindo a centralidade dessas tecnologias para manutenção da superioridade militar do país, conforme proposto pela third offset strategy, não obstante a mencionada redução do orçamento.

\section{CONSIDERAÇÕES FINAIS}

Este artigo identificou os vetores tecnológicos presentes na third offset strategy dos Estados Unidos, chamando atenção para a interdependência entre diferentes TIC e para a sua centralidade na condução de operações militares contemporâneas. A primeira seção tratou do processo histórico de digitalização das forças armadas estadunidenses e do desenvolvimento da noção de RMA que antecedeu a elaboração da offset strategy atualmente em curso. A segunda seção apresentou os fundamentos da estratégia e o objetivo estadunidense de manter um diferencial tecnológico em relação às potências emergentes no sistema internacional - notadamente China e Rússia - bem como em relação a ameaças assimétricas. Finalmente, a terceira seção tratou dos vetores tecnológicos presentes na estratégia: ciber; big data; nanotecnologia; armas de energia direta; sistemas espaciais; melhoramento humano; e robótica.

Essa centralidade crescente de TIC nas forças armadas, estimulada ainda pela maturidade tecnológica dos próprios sistemas, por forças de mercado e pelo avanço da digitalização, gera a necessidade de investigar como ocorre a incorporação dessas tecnologias pelos militares. O processo pode ser visto a partir de diferentes eixos, que englobam questões relativas desde ao estabelecimento de uma visão até o cumprimento de regras de engajamento internacionais, passando por elementos aparentemente tão desconexos como educação, doutrina, operações, etc. Perceber de que modo 
a tecnologia modifica cada um desses elementos, isolada e coletivamente, não é uma tarefa trivial e configura-se em um desafio importante, especialmente em contextos emergentes.

Ademais, processos de disseminação tecnológica e apropriação de prioridades estratégicas norte-americanas tornaram o desenvolvimento e a incorporação de novas tecnologias prioridade estratégica em diversas regiões. ${ }^{24}$ Soma-se a isso a necessidade de muitos países contrabalancearem o avanço dos Estados Unidos no sistema internacional por meio do fortalecimento e modernização de seus aparelhos militares. Esse processo de emulação, além de reforçar a lógica de dominação estadunidense na economia política mundial, pode ser danoso para países que buscam adequar suas forças armadas a partir de uma compreensão equivocada de seus próprios objetivos de segurança e defesa nacionais. No limite, a mera apropriação de percepções e de práticas exógenas pode resultar em um enfraquecimento do sistema político, das forças armadas, e na erosão da própria soberania estatal. Por outro lado, uma correta avaliação dos imperativos da third offset também pode proporcionar a esses países oportunidades de desenvolvimento de capacidades assimétricas que neutralizem, e quiçá esgotem, os avanços estadunidenses.

\section{REFERÊNCIAS}

Ackerman, Spencer. 2013. Budget Cuts are Set to Hit U.S. Military's Drone Fleet. Wired, [S.l.] (2 Apr.) https://www.wired.com/2013/04/drone-cuts/.

Akhgar, Babak. 2015. Application of Big Data for National Security: A Practitioner's Guide to Emerging Technologies. Oxford: Butterworth-Heinemann.

Armstrong, Benjamin. 2016. The Shadow of Air-Sea Battle and the Sinking of A2AD. War on the Rocks, [S.l.] (5 Oct). http://warontherocks.com/2016/10/the-shadow-of-air-sea-battle-and-the-sinking-of-a2ad/.

Arquilla, John. 2013. Could Killer Robots Bring World Peace? Foregin Policy, [S.l.] (19 Jun.) http://foreignpolicy.com/2013/06/19/could-killer-robots-bring-world-peace/.

Arquilla, John, and David Ronfeldt (Ed.). 1997. In Athena's Camp: Preparing for Conflict in the Information Age. Santa Monica: RAND.

Arrighi, Giovanni. 2008. Adam Smith em Pequim: Origens e Fundamentos do Século XXI. São Paulo: Boitempo. 
Axe, David. 2012. This Scientist Wants Tomorrow's Troops to Be MutantPowered. Wired, [S.l.] (26 Dec). https://www.wired.com/2012/12/andrew-herr/.

Bergen, Peter et al. 2016. World of Drones. New America Foundation, [S.l.]. https://www.newamerica.org/in-depth/world-of-drones/2-who-has-what-countries-drones-used-combat/.

Burgess, Matt. 2017. This Ridiculous Drone Gun can Shoot Down UAVs from $2 \mathrm{~km}$ Away. Wired, [S.l.] (23 Jan.) http://www.wired.co.uk/article/droneshield-dronegun-shoot-drone-uav-sky.

Burnam-Fink, Michael. 2011. The Rise and Decline of Military Human Enhancement. Science Progress, [S.l.] (7 Jan.). https://scienceprogress. org/2011/01/the-rise-and-decline-of-military-human-enhancement/.

Canabarro, Diego. 2014. Governança Global da Internet: Tecnologia, Poder e Desenvolvimento. Tese (Doutorado em Ciência Política) - Programa de PósGraduação em Ciência Política, Instituto de Filosofia e Ciências Humanas, Universidade Federal do Rio Grande do Sul, Porto Alegre.

Cebrowski, Arthur, and John Gartska. 1998. Network-Centric Warfare: Its Origins and Future. Proceedings Magazine [S.1.] 124.

Cepik, Marco et al. 2015. Espaço e Relações Internacionais. Porto Alegre: CEGOV.

Cepik, Marco, and Felipe Machado. 2011. O Comando do Espaço na Grande Estratégia Chinesa: Implicações para a Ordem Internacional Contemporânea. Carta Internacional 6 no. 2, 112-131.

Cohen, Eliot. 1999. American Views of the Revolution in Military Affairs. Mideast Security and Policy Studies, v. 28.

Coker, Christopher. 1998. Post-Modern War. RUSI Journal 143 no. 3, 7-14. http://www.tandfonline.com/doi/abs/10.1080/03071849808446260.

Cukier, Kenneth Neil, and Viktor Mayer-Schoenberger. 2013. The Rise of Big Data: How it's Changing the Way We Think about the World. Foreign Affairs [S.l.] (Mai./Jun.). https://www.foreignaffairs.com/articles/2013-04-03/rise-big-data.

Duarte, Érico. 2012. Conduta da Guerra na Era Digital e Suas Implicações para o Brasil: Uma Análise de Conceitos, Políticas e Práticas de Defesa. Rio de Janeiro: Ipea. 
Eaglen, Mackenzie. 2016. What is the Third Offset Strategy? Real Clear Defense [S.l.] (16 Feb.). http://www.realcleardefense.com/articles/2016/02/16/what_ is_the_third_offset_strategy_109034.html.

Ellman, Jesse, Lisa Samp, and Gabriel Coll. 2017. Assessing the Third Offset Strategy. Washington: Center for Strategic \& International Studies.

Farley, Robert. 2016. A2/AD Is Dead, Long Live A2/AD. The Diplomat [S.l.] (11 Oct.). http://thediplomat.com/2016/10/a2ad-is-dead-long-live-a2ad/.

Gettinger 2016

Gunzinger, Mark. 2013. Shaping America's Future Military Toward a New Force Planning Construct. Washington: Center for Strategic and Budgetary Assessments.

Gurin, Joel. 2014. Big Data and Open Data: What's What and Why Does It Matter? The Guardian, London (15 Apr.). https://www.theguardian.com/public-leaders-network/2014/apr/15/big-data-open-data-transform-government.

Hanlon, Mike. 2004 Future Warrior Suit 2020. New Atlas [S.l.] (20 Jan.). http:// newatlas.com/go/3062/.

Headrick, Daniel. 2009. Technology: A World History. Oxford: Oxford University Press.

Herr, Andrew. 2015. Will Humans Matter in the Wars of 2030? Joint Force Quarterly, v. 77, n. 2, 76-83. http://ndupress.ndu.edu/Media/News/NewsArticle-View/Article/581875/jfq-77-will-humans-matter-in-the-wars-of-2030/.

Howard, Michael. 1976. War in European History. Oxford: Oxford University Press.

IISS. 2015. The Military Balance 2015. Washington: International Institute for Strategic Studies.

Juengst, Eric. 1998. The Meaning of Enhancement. In: Parens, Erik. Enhancing Human Traits: Ethical and Social Implications. Washington: Georgetown University Press, 29-47.

Kelly, Kevin. 2007. The Technium and the $7^{\text {th }}$ Kingdom of Life. Edge [S.l.] (18 Jul.). https://www.edge.org/conversation/kevin_kelly-the-technium-and-the-7th-kingdom-of-life.

Kurzweil, Raymond. 2005. The Singularity is Near: When Humans Transcend Biology. New York: Penguin Press. 
Lawson, Sean. 2010. Is Network-Centric Warfare (Finally) Dead? Only Partly. ICTS and International Affairs [S.l.] (14 Aug.). http://www.seanlawson.ne$\mathrm{t} / \mathrm{P} \mathrm{p}=772$.

Leonhard, Robert. 1998. The Principles of War for the Information Age. Novato: Presidio Press.

Libicki, Martin. 2012. Cyberspace is not a Warfighting Domain. I/S: A Journal of Law and Policy for the Information Society 8 no. 2, 325-340. http://moritzlaw.osu. edu/students/groups/is/files/2012/02/4.Libicki.pdf.

Lin, Patrick. 2013. Could Human Enhancement Turn Soldiers Into Weapons That Violate International Law? Yes. The Atlantic [S.l.] (4 Jan.). http://www. theatlantic.com/technology/archive/2013/01/could-human-enhancement-turn-soldiers-into-weapons-that-violate-international-law-yes/266732/.

Lin, Patrick et al. 2013. Super Soldiers (Part 1): What is Military Human Enhancement? In: Steve John Thompson. Global Issues and Ethical Considerations in Human Enhancement Technologies. Hershey : IGI Global, 119-138.

Lonsdale, David. 2004.The Nature of War in the Information Age: Clausewitzian Future. London: Frank Cass.

Macgregor, Douglas. 2012. Building a Smarter, Smaller Military. Time [S.l.] (6 Dec.). http://nation.time.com/2012/12/06/building-a-smarter-smaller-military/.

Mann, Adam. 2013. Google's Chief Internet Evangelist on Creating the Interplanetary Internet. Wired, [S.l.] (6 Mai). https://www.wired.com/2013/05/ vint-cerf-interplanetary-internet/.

Mcneil, William. 1982. The Pursuit of Power: Technology, Armed Forces and Society since A.D. 1000. Chicago: The University of Chicago Press.

Mavani, Kandarp; Shah, Mihir. 2013. Synthesis of Silver Nanoparticles by using Sodium Borohydride as a Reducing Agent. International Journal of Engineering Research \& Technology (IJERT), v. 2, n. 3. https://www.ijert.org/research/synthesis-of-silver-nanoparticles-by-using-sodium-borohydride-as-a-reducing-agent-IJERTV2IS3605.pdf.

Nasu, Hitoshi. 2013. The Future of Nanotechnology in Warfare. The Global Journal, [S.l.] (4 Jul.) http://www.theglobaljournal.net/article/view/1132/.

Possamai, Ana Júlia. Dados Abertos no Governo Federal Brasileiro: Desafios de Transparência e Interoperabilidade. 2016. Tese (Doutorado em Ciência Política) - 
Instituto de Filosofia e Ciências Humanas, Programa de Pós-Graduação em Ciência Política, Universidade Federal do Rio Grande do Sul. Porto Alegre, 2016.

Richardson, John. 2016. Chief of Naval Operations Adm. John Richardson: Deconstructing A2AD. The National Interest [S.l.] (3 Oct.). http://nationalinterest.org/feature/chief-naval-operations-adm-john-richardson-deconstructing-17918

Rid, Thomas. 2013. Cyber War Will not Take Place. Oxford: Oxford University Press.

Roland, Alex. 1997. Technology and War. Chapel Hill: American Diplomacy. http://www.unc.edu/depts/diplomat/AD_Issues/amdipl_4/roland.html\#intro.

2009. War and Technology. Philadelphia: Foreign Policy Research Institute. http://www.fpri.org/article/2009/02/war-and-technology.

2016. War and Technology: A Very Short Introduction. Oxford: Oxford University Press.

Schachtman, Noah. 2004. Darpa Offers No Food for Thought. Wired, [S.l.] (17 Feb.) https://www.wired.com/2004/02/darpa-offers-no-food-for-thought/.

Schachtman, Noah. 2012. U.S. Spies See Superhumans, Instant Cities by 2030. Wired, [S.l.] (12 Oct.) https://www.wired.com/2012/12/superhumans-instant-cities/

Singer, Peter. 2009. Wired for War: The Robotics Revolution and Conflict in the $21^{\text {st }}$ Century. New York: Penguin Press.

Siniscalchi, Joseph. 2001. Nonlethal Technologies and Military Strategy. In Martel, William (Ed.). The Technological Arsenal: Emerging Defense Capabilities. Washington: Smithsonian Institute, 129-152.

Stulberg, Adam, and Michael Salomone. 2007. Managing Defense Transformation: Agency, Culture and Service Change. Hampshire: Ashgate.

Symon, Paul, and Arzan Tarapore. 2015. Defense Intelligence Analysis in the Age of Big Data. Joint Force Quarterly 79, no. 4, 4-11. http://ndupress.ndu.edu/ Media/News/News-Article-View/Article/621113/defense-intelligence-analysis-in-the-age-of-big-data/.

Toffler, Alvin, and; Heidi Toffler, 1997. Foreword: The New Intangibles. In Arquilla, John, and David Ronfeldt, (Ed.). In Athena's Camp: Preparing for Conflict in the Information Age. Santa Monica: RAND, XIII-XXIV. 
Turse, Nick. 2016. Pentagon Video Warns Of "Unavoidable" Dystopian Future For World's Biggest Cities. The Intercept [S.l.] (13 Oct.). https://theintercept. com/2016/10/13/pentagon-video-warns-of-unavoidable-dystopian-future-for-worlds-biggest-cities/.

Van Creveld, Martin. 1991a. Technology and War: From 2000 B.C. to the Present. New York: The Free Press.

Van Creveld, Martin. 1991b. The Transformation of War: The Most Radical Reinterpretation of Armed Conflict since Clausewitz (A Revised and Expanded Edition). New York: The Free Press.

Vanderbilt, Tom. 2004. The Real da Vinci Code. Wired [S.l.] (Nov.). http://archive. wired.com/wired/archive/12.11/davinci.html?pg=1\&topic=davinci\&topic_set.

Verbeek, Peter-Paul, and Pieter Vermass. 2009. Technological Artifacts. In Olsen, Jan, Andur Pedersen, and Vincent Hendricks (Ed.). A Companion to the Philosophy of Technology. Oxford: Blackwell, 165-171.

Wang, Brian. 2016. Russia Indicates that They are Matching US Hard and Soft Military Exoskeleton Development. Next Big Future [S.l.] (15 Nov.). http://www. nextbigfuture.com/2016/11/russia-indicates-that-they-are-matching.html.

Wong, Wilson. 2013. Emerging Military technologies: A Guide to the Issues. Santa Barbara: Praeger.

Work, Robert. 2016. Remarks by Deputy Secretary Work on Third Offset Strategy as Delivered by Deputy Secretary of Defense Bob Work, Brussels, Belgium, April 28, 2016. U.S. Department of Defense [S.l.]. https://www.defense.gov/News/ Speeches/Speech-View/Article/753482/remarks-by-d\%20eputy-secretary-work-on-third-offset-strategy.

World Bank. 2017. Armed Forces Personnel, Total [S.1]. http://data.worldbank. org $/$ indicator $/$ MS.MIL.TOTL.P 1 ?end $=2015 \& \quad$ locations $=$ US\&page $=4 \&$ star$\mathrm{t}=1985$ \&view $=$ chart.

Wright, Quincy. 1942a. A Study of War Vol. 1. Chicago: University of Chicago Press.

Wright, Quincy. 1942b. A Study of War Vol. 2. Chicago: University of Chicago Press.

Young, Chris. 2012. Military Intelligence Redefined: Big Data in the Battlefield. Forbes, [S.l.], (12 Mar.) http://www.forbes.com/sites/techonomy/2012/03/12/ military-intelligence-redefined-big-data-in-the-battlefield/\#20fbe2a6718f. 


\section{NOTAS}

1. Logo após a Primeira Guerra Mundial, o cientista político Quincy Wright e seus colegas da Universidade de Chicago deram início a um projeto de pesquisa interdisciplinar cujo objetivo era estudar as causas da guerra. O projeto resultou em mais de cinquenta publicações, incluindo a principal obra do autor, "A Study of War" (1942). Em seu livro, Wright desenvolveu um modelo evolutivo da guerra baseado em quatro estágios (animal, primitivo, civilizado, moderno), cada qual amparado por um agente causal (indefinido, sociedade, sistema internacional, tecnologia) e por uma disciplina explanatória distinta (psicologia, sociologia, direito internacional, ciência). A transição de um estágio para o outro, segundo Wright, foi causada por mudanças nas formas de comunicação: falada, escrita e imprensa, respectivamente. Apesar de datado - e, segundo a crítica de Roland (1997), extremamente determinista por sugerir que a tecnologia é a causa da guerra moderna —, o modelo de Wright proveu uma base sólida para a discussão sobre guerra e tecnologia que o procederia.

2. $\mathrm{O}$ autor sugere um experimento mental interessante para amparar a sua tese: seria Alexandre, o Grande, capaz de conquistar o Afeganistão novamente no século XXI? Para Roland (2009; 2016) ainda que Alexandre dominasse os princípios fundamentais e imutáveis da guerra - definidos para o autor a partir de uma visão jominiana como objetivo, ofensiva, concentração de força, economia de força, manobra, unidade de comando, segurança, surpresa e simplicidade - , o mesmo não conseguiria aplicar as tecnologias disponíveis na atualidade para alcançar os seus objetivos militares. A razão da sua incapacidade seria justamente as mudanças tecnológicas ocorridas desde o século IV a.C. e as decorrentes transformações no caráter do fenômeno.

3. Ademais, as forças armadas passaram a contar também com um número crescente de técnicos - grosso modo, analistas de sistemas responsáveis por operar tais computadores. Os mesmos, segundo Van Creveld (1991), introduziram nas forças armadas, especialmente dos Estados Unidos, a percepção de que seria possível quantificar a prática da guerra por meio de modelos matemáticos. Neste sentido, a visão avançada por essa nova tecnocracia teria contribuído para a derrota do país no Vietnã, sobretudo por desconsiderar fatores irracionais (psicológicos) e/ou não-racionais (naturais) no desenvolvimento do conflito. Paralelamente, começou-se a construir nos Estados Unidos o que o ex-presidente Dwight Eisenhower chamou de "complexo militar-industrial”, com o objetivo de institucionalizar a prática de pesquisa e desenvolvimento de novas tecnologias. Nas palavras de Roland (2009, 5), 
"the introduction of systematic, institutionalized innovation makes modern military technology seem radically different from all that went before". Dessa forma, diferentemente do que ocorria até antes da Segunda Guerra Mundial, quando um militar provavelmente passaria toda a sua carreira operando com os mesmos instrumentos, desde meados do século XX é cada vez mais difícil afirmar que tecnologia será empregada na próxima guerra.

4. Vale ressaltar, contudo, que a Era Digital não diz respeito apenas a computadores e dados digitais. Nas palavras de Alvin Toffler e Heidi Toffler (1997, XIV), no cerne da revolução digital "lies a shift in the relationship between tangible and intangible methods of production and destruction alike". Nesse sentido, "[knowledge $]$ has moved from a peripheral to a central position”. Por isso, na visão dos autores, ideias, valores, símbolos, e imagens importam tanto quanto dados computacionais. Em termos militares, a relevância de aspectos imateriais e/ou intangíveis está tradicionalmente vinculada a questões como moral, liderança, coragem, etc. Mais recentemente, ela passou a estar relacionada também à capacidade de infringir dano por meio de ações perpetradas no chamado ciberespaço.

5. Segundo dados do Banco Mundial (2016), os gastos militares estão mais ou menos estáveis tanto nos Estados Unidos (entre 3\% e 4,5\% do PIB) quanto na Rússia (entre 3,5\% e 4\% do PIB) desde o início dos anos 2000. Os dados do Stockholm International Peace Research Institute (SIPRI) (2016) corroboram essa informação. Os dados da SIPRI também indicam, contudo, uma queda acentuada nos gastos militares de ambos os países entre 1990 e 2000: de 5,7\% para 3\% do PIB nos Estados Unidos, e de $15,8 \%$ para $3,6 \%$ do PIB na União Soviética/ Rússia. Recentemente, constrangimentos notadamente econômicos sobre as forças armadas intensificaram-se graças à crise fiscal que se instalou sobre diversos países a partir de 2008. Nesse sentido, muitos economistas sugerem que crises econômicas favorecem a inovação tecnológica e o desenvolvimento de processos de produção mais eficientes, uma vez que permitem a alocação mais racional de recursos limitados. Em outras palavras, tempos de crise ajudariam os Estados a determinarem suas prioridades, apontando para o desenvolvimento de algumas capacidades críticas em detrimento de outras menos relevantes (Macgregor 2012).

6. O descontentamento com a política externa estadunidense foi intensificado na década de 1990, também em função do chamado "efeito CNN". A cobertura midiática intensa da Guerra do Golfo, transmitida ao vivo para o mundo, não apenas retroalimentou os preceitos da RMA, dis- 
cutida em detalhes na próxima nota, como também contribuiu para aumentar a desconfiança internacional a respeito das pretensões norte-americanas no sistema internacional. Nesse sentido, a própria RMA parece ter despertado uma série de lideranças internacionais para a necessidade de investir em um processo de catch up.

7. Aliada a esta crescente dependência computacional está o surgimento da Internet como plataforma de convergência para diversas atividades sociais. Ainda que seu desenvolvimento tenha ocorrido paralelamente à criação de outras soluções para interoperação de computadores, algumas características intrínsecas da Internet fizeram dela, ainda no final do século passado, a rede das redes. Dentre as características que ajudaram a Internet a vencer os modelos concorrentes disponíveis então, destacam-se, por exemplo, a possibilidade de emprego de padrões abertos e gratuitos, a estruturação aberta e participativa de sua governança técnica e sua estruturação como plataforma neutra, sem controle centralizado sobre os tipos de aplicações e funcionalidades desenvolvidos para rodar sobre ela (Canabarro 2014). A centralidade da Internet e sua importância para os processos de digitalização fomentaram a criação de um subcampo dos estudos de segurança voltado justamente para a investigação de assuntos relacionados ao ciberespaço. Além disso, estimularam também a realização de estudos sobre mobilização política através de plataformas sociais digitais, evidenciando, assim, algumas mudanças fundamentais na forma com a qual nos relacionamos com a tecnologia. Isso, por sua vez, impacta decisivamente a relação entre o Estado e seus cidadãos, bem como a relação entre atores estatais e não estatais nos termos dos principais debates das teorias das Relações Internacionais consolidadas ao longo do século XX. Uma parcela cada vez mais significativa da literatura tem atribuído implicações revolucionárias ao desenvolvimento e propagação de TIC. Em geral, estes trabalhos preveem o empoderamento de atores não-estatais, o declínio do Estado-nação, a irrelevância do mundo material e a crescente importância de dados eletrônicos às expensas de capacidades militares tradicionais. Tais previsões carecem, quando da confecção deste trabalho, de suporte empírico inequívoco e amplamente aceito.

8. Para muitos autores, a emergência da Era Digital e de todas as tecnologias atreladas a ela apenas reforça a ideia de que estamos na iminência da singularidade. A singularidade tecnológica refere-se a um momento histórico futuro no qual a inteligência artificial terá superado a inteligência humana, alterando radicalmente a civilização e a própria natureza do ser humano (Kurzweil 2005). 
9. Naturalmente, prever as tecnologias que serão determinantes para as guerras futuras é uma tarefa complicada, sobretudo se consideramos a ideia comum entre os militares de que "nos preparamos para lutar a última guerra”. Por isso, o estabelecimento de objetivos de defesa bem definidos é fundamental, conforme veremos no capítulo sobre desenho de força.

10. A complexidade dos ambientes operacionais do século XXI foi abordada recentemente em um vídeo produzido pela Universidade de Operações Especiais Conjuntas do Pentágono dos Estados Unidos e divulgado pelo canal alternativo The Intercept. Mais especificamente, o vídeo trata do papel das megacidades, descritas como "complex systems where people and structures are compressed together in ways that defy both our understanding of city planning and military doctrine”. Segundo o narrador da peça, "these are the future breeding grounds, incubators, and launching pads for adversaries and hybrid threats" (Turse 2016).

11. Recentemente, alguns autores começaram a questionar a utilidade do conceito de A2AD. O debate foi iniciado pelo Almirante John Richardson, Chefe de Operações Navais da Marinha dos Estados Unidos. Segundo ele, "to some, A2AD is a code-word, suggesting an impenetrable 'keep-out zone' that forces can enter only at extreme peril to themselves. To others, A2AD refers to a family of technologies. To still others, a strategy. In sum, A2AD is a term bandied about freely, with no precise definition, that sends a variety of vague or conflicting signals, depending on the context in which it is either transmitted or received" (Richardson 2016). Para o Almirante, o termo deve ser abandonado pela Marinha dos Estados Unidos justamente devido a esta falta de clareza conceitual. Além disto, já que a prática não é completamente nova, dedicar mais esforços para a sua compreensão é desnecessário. De acordo com ele, a Marinha já tem pleno entendimento do fenômeno, de modo que “the Navy's focus must remain on maintaining maritime superiority, with a deep understanding of the interplay between tactics and strategy, against specific threats, in specific locations, to achieve that end".

As declarações do Almirante causaram reações variadas. Para Robert Farley (2016), por exemplo, o termo "may effectively represent a particular manifestation of a long-running strategic problem. This manifestation is characterized by the threat of a previously unachievable degree of coordination between different tools of national defense, geared towards winning freedom of movement in a constrained maritime space”. O texto de Benjamin Armstrong (2016), por sua vez, vai ao encontro da crítica de Richardson (2016), ao afirmar que a criação 
de "buzzwords" como A2AD não tem "the potential to hurt naval thinking more than they help it".

12. Neste trabalho, o emprego de C3I é precedido de "sistemas computadorizados", indicando a presença deste componente na cadeia.

13. Uma premissa auxiliar, presente em parte da literatura, é a de que as guerras futuras serão mais violentas e/ou letais do que as passadas. Consequências dessa premissa incluem, por exemplo, a possibilidade de que guerras interestatais sejam menos recorrentes no futuro em virtude do papel dissuasório de sistemas robóticos. John Arquilla (2013), por exemplo, colocou a hipótese da seguinte forma: "the cool, lethal effectiveness of robots properly used might, just might, give potential aggressors pause, keeping them from going to war in the first place. For if invading human armies, navies, and air forces can be decimated by defending robots, the cost of aggression will be seen as too high. Indeed, the country, or group of countries, that can gain and sustain an edge in military robots might have the ultimate peacekeeping capability".

14. A estratégia faz uma seleção um pouco distinta, identificando seis áreas-chave: A2AD; munições guiadas; guerra submarina; guerra cibernética e eletrônica; pareamento homem-máquina (human-machine teaming); e simulações (wargaming) e desenvolvimento de novos conceitos operacionais. Segundo Mackenzie Eaglen (2016), "in 2017, the budget contains at least $\$ 1$ billion in anti-access and area denial spending, $\$ 489$ million in guided munitions spending, \$508 million in undersea warfare spending, \$201 million in human-machine teaming, \$309 million in cyber and electronic warfare, and $\$ 155$ million in wargaming and new operational concept development".

15. Naturalmente, alguns problemas como analfabetismo e exclusão digital também são frutos desse espalhamento da Internet. Infelizmente, analisar mais especificamente os resultados sociais da Era Digital fogem do escopo deste trabalho.

16. Não apenas para as forças armadas, mas também para os indivíduos e para o próprio provimento de segurança pública e segurança nacional.

17. Além, naturalmente, dos instrumentos de monitoramento e vigilância mais tradicionais. Neste sentido, a relação entre UAS e big data é interessante e foi explorada em artigo de Terry Costlow (2014).

18. Nesse contexto, o conceito de "open data", ou dados abertos, torna-se também relevante. Segundo Joel Gurin (2014), “open data brings a perspective that can make big data more useful, more democratic, and less threatening". De acordo com o pesquisador, é importante relacionar "dados abertos" e "big data" já que ambos "can transform business, 
government, and society - and a combination of the two is especially potent. Big data gives us unprecedented power to understand, analyse, and ultimately change the world we live in. Open data ensures that power will be shared - and that the world we change will, with luck, become a fairer and more democratic one". O autor ainda chama atenção para a relevância do conceito de "governo aberto" nesse mesmo contexto. Infelizmente, o aprofundamento dessas questões foge do escopo deste trabalho. Por isso, recomenda-se aos interessados a leitura de Possamai (2016).

19. Apesar das dificuldades técnicas envolvidas no desenvolvimento de armas de energia direta de emprego militar, alguns modelos, de alcance reduzido, já estão disponíveis no mercado, conforme aponta a seguinte matéria publicada por Matt Burgess (2017).

20. O próprio Vincent Cerf já projetou a possibilidade de se criar uma "Internet interplanetária". Em entrevista para revista "Wired" (Mann 2013), Cerf afirmou que: "up until that time and generally speaking, up until now, the entire communications capabilities for space exploration had been point-to-point radio links. So, we began looking at the possibilities of TCIP/IP as a protocol for interplanetary communication”. Naturalmente, conectar diferentes planetas por meio da internet é uma tarefa que enfrenta diversos desafios e limitações comuns a qualquer tipo de operação no espaço sideral.

21. No contexto da third offset, o debate sobre a criação de um comando espacial independente, conforme proposta do atual presidente estadunidense, denota justamente a preocupação do país em manter-se como hegêmona espacial frente às potências emergentes.

22. É interessante notar que, diferentemente de outras categorias que no limite preveem a completa substituição de seres humanos por tecnologias, o melhoramento humano entende que aqueles sempre terão um papel fundamental na guerra. Nesse sentido, vale citar a seguinte passagem de Andrew Herr (2015, 77) sobre as relações entre homens e computadores: "today's scientific and technological landscape suggests that the human brain will still substantially outperform computers in the highest level cognitive tasks in 2030. Furthermore, the competition is not simply between the brain and computers, but rather between computers and humans augmented by computers".

23. Um exemplo é o projeto da DARPA intitulado "Metabolic Dominance". Mais detalhes sobre a iniciativa podem ser encontrados em Shachtman (2004). Vale apena mencionar ainda o papel que componentes gerados a partir de impressoras 3D, inclusive órgãos artificiais, pode desempenhar na área do melhoramento humano (Shachtman 2012). 
24. Esta tendência de homogeneização - ou de tentativa de homogeneização - das capacidades tecnológicas de diferentes países é válida, grosso modo, para todo o tipo de tecnologia. O fenômeno parece ser ainda mais claro quando diz respeito a tecnologias militares: "the introduction of a weapon by one military power very often serves as the justification par excellence for its acquisition by all the rest, modern military technology tends towards homogeneity" (Van Creveld 1991, 290). 
TECNOLOGIAS MILITARES EMERGENTES:

\section{DIGITALIZAÇÃO E A THIRD OFFSET STRATEGY ESTADUNIDENSE}

\section{RESUMO}

Com o final da Guerra Fria, a digitalização das forças armadas se tornou a ordem do dia nos Estados Unidos. Desde então, o país vem investindo no desenvolvimento de uma série de vetores tecnológicos que prometem preservar sua supremacia militar e manter o seu papel de destaque nas relações internacionais nas próximas décadas. $\mathrm{O}$ presente artigo tem caráter exploratório. Nesse sentido, busca identificar esses vetores e analisar as possíveis implicações que seu desenvolvimento e adoção poderão trazer para as forças armadas norte-americanas. Destaca-se, assim, a importância de tecnologias ciber; big data; nanotecnologia; armas de energia direta; sistemas espaciais; melhoramento humano; e robótica para o desenvolvimento das capacidades estadunidenses. Conclui-se que o processo de offset traz consigo uma necessidade de reflexão profunda, especialmente entre países emergentes, a respeito de suas próprias capacidades e oportunidades de modernização, sem que isso resulte, necessariamente, na emulação do processo estadunidense.

Palavras-chave: Digitalização; Tecnologia; Third Offset Strategy.

\section{ABSTRACT}

Digitization has become the order paper for the U.S. military since the end of the Cold War. The country has been investing ever since in the development of a series of technological vectors (cyber, big data, nanotechnology, direct energy weapons, space systems, human enhancement, and robotics) that promise to maintain America's military supremacy and leading role in the international system in the upcoming decades. This paper has an exploratory character. It aims at identifying the aforementioned vectors and analyzing the implications brought about by the incorporation of such technologies to the U.S. military. As a final remark, the paper stresses the need for every country willing to modernize their armed forces to reflect upon their own necessities without necessarily emulating America's strategy.

Keywords: Digitization; Technology; Third Offset Strategy. 